\title{
Mathematical Modeling of Covid-19 Disease Dynamics and Analysis of Intervention Strategies
}

\author{
Rotich Kiplimo Titus ${ }^{1,}$, , Lagat Robert Cheruiyot ${ }^{2}$, Choge Paul Kipkurgat ${ }^{1}$ \\ ${ }^{1}$ Department of Mathematics Physics and Computing, Moi University, Eldoret, Kenya \\ ${ }^{2}$ Department of Mathematics and Actuarial Science, South Eastern Kenya University, Kitui, Kenya
}

Email address:

tisesko@yahoo.com (R. K. Titus), lagatrobert9@gmail.com (L. R. Cheruiyot), chogepaul0@gmail.com (C. P. Kipkurgat)

${ }^{*}$ Corresponding author

\section{To cite this article:}

Rotich Kiplimo Titus, Lagat Robert Cheruiyot, Choge Paul Kipkurgat. Mathematical Modeling of Covid-19 Disease Dynamics and Analysis of Intervention Strategies. Mathematical Modelling and Applications. Vol. 5, No. 3, 2020, pp. 176-182. doi: 10.11648/j.mma.20200503.16

Received: July 17, 2020; Accepted: July 29, 2020; Published: August 10, 2020

\begin{abstract}
Covid-19 is a highly prevalent contagious disease, with high fatalities. With the absence of a one bullet drug or vaccine, infected individual is highly likely to die within a short time. The transmission and progression of Covid-19 can be described using distinct stages, namely exposure and latency, infectiousness, and recovery with waning immunity or death. This implies that, mathematical model will place individuals into four compartments, that is, Susceptible (S), Exposed (E), Infective (I) and Recovered (R), representing a SEIR model. Due to its fast fatal capacity, changes in population due to births do not affect the disease dynamics, but for the purpose of monitoring deaths, a compartment for deaths (D) is incorporated. The analysis of intervention strategies necessitates modification of SEIR model to include Quarantine (Q), Isolation (I), and Homebased care $(\mathrm{H})$ compartments. In this paper, Public health Education Campaign, Quarantine and testing, Isolation, Treatment, use of facemask and Social distance intervention strategies were analyzed. Numerical results indicated that the most responsive mitigation strategy is use of quality facemask and observance of social distance. At $90 \%$ adherence to this plan reduces the force of infection from $\beta=0.0197$ to $\beta=0.0033$. This will consequently reduce the basic reproductive ratio from $R_{0}=14.0362$ to $R_{0}=2.3388$, which prevents $99.37 \%$ of population from contracting the disease. However, it is shown that a combination of other intervention strategies have synergetic effect of bringing down reproductive ratio to less than one. Sensitivity analysis indicated that isolation and treatment of infected individuals in government facilities is the most effective method with elasticity of $v=-6.4$, but due to financial implications, the alternative homebased care need to be fortified. This means, for Covid-19 pandemic to die off, we require early identification of infected individuals through mass testing and immediate isolation. In order to optimize financial constraints associated with isolation, currently at $\alpha=11 \%$, the threshold efficacy of other intervention strategies should be enhanced to; public health campaign $\epsilon>50 \%$, complacency $\xi<30 \%$, facemask quality c $>89 \%$, social distance $r>2 \mathrm{~m}$, and mass testing $\tau>0.27$. With these interventions, it is estimated that the reproductive ratio, reduces to less than one after 225 days from the first occurrence of Covid-19, and the epidemic will then begin to decline gradually to insignificant levels.
\end{abstract}

Keywords: Face mask Efficacy, Radial Social Distance, Reproductive Ratio, Complacency, Isolation, Homebased Care

\section{Introduction}

The 2019 Novel Coronavirus $(2019 \mathrm{nCoV})$, is a new zoonotic pathogen in the family of SARS-CoV and MERS$\mathrm{CoV}$ among others [1]. This was named in February, 2020 as Severe Acute Respiratory Syndrome Coronavirus 2 (SARSCoV-2), with associated disease officially named as Covid-19 [2].
The name Coronavirus was first coined in 1968 derived from the 'crown-like' morphology as observed in an electron microscope [3]. When infected, the virus causes severe acute respiratory syndrome, characterized by coughing, sneezing, difficulty in breathing and high fever. The disease in not airborne, but transmitted through oral fluids (sputum) as a result of coughing or sneezing by an infected person. Once a person is exposed to the virus, and successfully infected, the victim remains latent for short period, then become 
symptomatic. It has however been observed that even while at latency, the asymptomatic individual is able to transmit the disease. There is no effective regimen available currently, but symptoms are managed using antibiotics and fever relief drugs.

Among many intervention strategies, the most advocated Covid-19 disease control method is through prevention of spread through the use of face masks, hygiene, social distancing, quarantine, lockdown among others.

Corona Virus pandemic has caused immense negative impacts on every sector in the world, ranging from economy, education, political, spiritual, name it got it. Because of this effect, every individual in the world is now trying to find any possible way on how to contain or eradicate Covid-19. Some of the methods people use include provision of hand washing stations, fumigation of working surfaces, use of hand sanitizers, production of face masks, local design and development of ventilator, opening up of quarantine centers, public health sensitization campaigns among others. As much as the medical research scientists, epidemiologists, pharmacist, nurses and doctors are in the forefront in the fight against Covid-19, mathematicians are not left out either.

Mathematical epidemiology is a branch of biomathematics which uses mathematical models to describe the current and future dynamics of disease spread, analysis of intervention strategies efficacies, and propose through simulation the optimal thresholds of each strategy in relation to cost, space and time.

In order to use mathematics in modelling diseases, individuals are classified basing on their distinct epidemiologic characteristics into compartments. At the onset of the disease, the entire population is susceptible $(\mathrm{S})$. When exposed to the virus, they are moved to Exposed (E) or Latent compartment. The disease then progresses to symptomatic and Infectious (I) stage, forming an infectious class. After this, individuals seek treatment, where they recover fully or die. The compartment holding the recovered class is denoted by $(\mathrm{R})$. Some diseases however confer immunity upon infection. If immunity is permanent, a new compartment is created called Immune (M), otherwise temporary immunity will lead to recoveries reverting back to Susceptible class. Common compartmental paradigms used include; SIS, SIR, SEIR, SEIRS, SITA, among others [4, 5].

In the case of Covid-19, the most relevant model is SEIR, where from susceptible, individuals become exposed, then progress to infective and after seeking treatment, recovers. The flow chart representing this model is presented in Figure 1 below. In this case, demographic factors are not considered, because of the short term progression of the disease. Infection induced mortality is the only one accounted in the infective class.

Susceptible class generally represents the entire population who can get infected. Since the disease is fast spreading, and can show symptoms within a short period, and due to limitation in testing capacity, the term susceptible in our model represents the sampled size for testing. Recruitment into this class represents the testing rate. This will change in the long term, when majority of the population will have been tested.

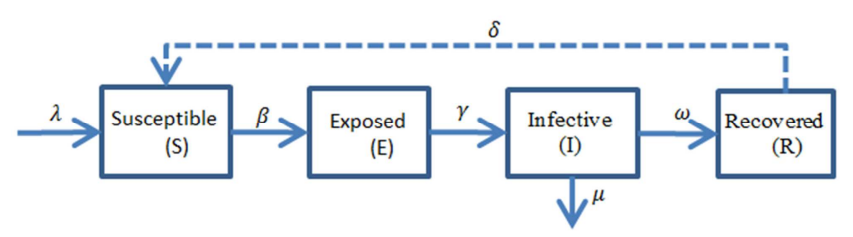

Figure 1. Model Flow Chart of Covid-19 Disease Dynamics.

Note that, the flow chart in Figure 1. above has a dotted line joining the recovered individuals back to the susceptible. This represents the proportion that become re-infected upon waning of temporary immunity acquired after the first infection. The model equations are given by;

$$
\begin{aligned}
& \mathrm{S}^{\prime}=\lambda-\beta S I+\delta R \\
& \mathrm{E}^{\prime}=\beta S I-\gamma E \\
& \mathrm{I}^{\prime}=\gamma E-(\omega+\mu) I \\
& \mathrm{R}^{\prime}=\omega I-\delta R
\end{aligned}
$$

where; $\lambda$ is the testing rate, $\beta$ is the probability of getting infected after sufficient contact with infective, $\delta$ is the diminishing rate of temporary immunity acquired, $\omega$ the recovery rate, $\gamma$ is the progression rate from exposed to infective and $\mu$ is the disease related death rate.

The model in equation $(1-4)$ is common to many fast spreading infectious diseases, whose dynamics cannot be affected by natural death and birth rates. This applies to Covid-19 too, but modification is required to capture additional dynamics witnessed by Covid-19.

\section{Model Formulation}

Much epidemiological information is developing about Novel Coronavirus $(2019 \mathrm{nCoV})$. These include reinfection possibility, mitigation strategies and development of treatment regimen. The world as a whole uses prevention techniques to curb the spread, and minimize the burden of overwhelmed medical facilities.

Mathematical models are therefore relevant in describing the dynamics and determining the threshold efforts for each strategy, in order to optimize the limited resources. In this paper, mitigation strategies are analyzed to shed light on where and how much containment efforts is to be directed. In order to do this, additional compartments are introduced to capture every classification of individuals with respect to Covid-19 disease and all possible control strategies.

\subsection{Model Variables and Parameters}

The mathematical model variables are; $S_{h}(t), S_{l}(t), E(t), I(t), R(t), Q(t), J(t), H(t)$ and $D(t)$ for High risk Susceptible, Low risk Susceptible, Exposed, Infective, Recovered, Quarantined (for testing and transmission to Isolation), Isolated (in government facility or 
hospital due to acute status of infection), Homebased care, (isolated and taking treatment from home) and Deaths respectively, with a constant total population $S_{\mathrm{h}}+\mathrm{S}_{\mathrm{l}}+E+$ $I+R+Q+J+H+D=N$.

It is here assumed that, due to public health campaigns, individuals become educated, and practice low risk behavior which include; use of mask, avoiding public gathering, observing social distancing, frequently washing hands, limitation of movement (lock down and curfew), closing of schools, markets and churches, working from home among others. These people are classified as Low risk susceptible $\left(S_{l}(t)\right)$. On the other hand, those who work in the hospitals, the care givers, those who travel, truck drivers, essential service providers, those not observing WHO regulations [6] fall under High risk Susceptible $\left(S_{h}(t)\right)$. Due to pressure in government facilities, asymptomatic patients as recommended by a doctor, can take treatment and cared from home. This creates additional isolation center as Homebased care $(H(t))$, while the complement in government isolation facilities is $(J(t))$. As the statistics of the dead is required, our model appends additional compartment for the dead as $(D(t))$.

\subsection{Model Parameters}

With these variables, the parameters in Table 1 . below, were used to formulate the mathematical model.

Table 1. Covid-19 Mathematical Model Parameters.

\begin{tabular}{|c|c|c|}
\hline Symbol & Description & Source \\
\hline$\alpha$ & Proportion of positively tested people to government isolation canter for treatment & Variable $[0,1]$ \\
\hline$\hat{\beta}$ & Probability of getting infected due to sufficient interaction with an infected person & Constant \\
\hline$\epsilon$ & Change of behaviour due to public health education campaign on prevention of Covid-19 & Variable $[0,1]$ \\
\hline$\delta$ & Reduced rate of infection by exposed asymptomatic individuals & Constant \\
\hline$\sigma$ & Rate of return to high risk susceptible after testing negative & Assumed \\
\hline$\mu$ & Accelerated death rate due to Covid-19 infection & Constant \\
\hline$\rho$ & Loss of immunity and returning to susceptible class for re-infection & Assumed \\
\hline$\eta$ & Transfer rate of exposed asymptomatic class for testing and quarantine (Testing rate) & Constant \\
\hline$\phi$ & Effect of isolation and treatment in reducing death rate & Constant \\
\hline$\psi$ & Transfer rate of infected symptomatic individuals to testing and quarantine class & Variable $[0,1]$ \\
\hline$\theta$ & Reduction of rate of recovery due to lack of standards in home based care & Assumed \\
\hline$\omega$ & Recovery rate of infected individuals & Constant \\
\hline$\gamma$ & Progression rate of exposed individuals to infective stage & Constant \\
\hline$\xi$ & Complacency rate, where individuals revert to old normal & Variable $[0,1]$ \\
\hline$\vartheta$ & Modified (reduced) infective rate of exposed asymptomatic individuals & Constant \\
\hline$\kappa$ & Modification of infectivity by homebased infected individuals & Constant \\
\hline$\partial$ & Rate of transfer of home based to Isolation & Variable $[0,1]$ \\
\hline$\varpi$ & Transfer of individuals to low risk after testing negative & Constant \\
\hline$c$ & Viral particles transmissivity constant & Constant \\
\hline$m$ & Face mask Porosity & Variable $[0,1]$ \\
\hline$d$ & Social distance observed between an infective and a susceptible individual & Variable $[0, \infty)$ \\
\hline
\end{tabular}

\subsection{Model Assumptions}

Together with the variables and parameters, the following assumptions about the disease and the population are made.

The entire population is homogeneous, and can contract the disease equally, regardless of age, race, gender, place of residence, profession, or level of immunity.

The parameters are deterministic and their effect on variables is instantaneous, and not affected by environmental or demographic factors.

The recovered class represents those who are treated effectively and test negative.

Once an individual recover, the disease confers temporary immunity, which wanes with time.

The disease dynamics are so fast that the effect of death and birth demographics is insignificant.

The quarantined class represents those who are undergoing tests, and can test positive or negative. This includes those who have recently travelled from highly infected areas, or those with fever but not yet confirmed to be infected by Covid-19.

Isolated cases are those confirmed to be infected. They could be asymptomatic or mild, thus recommended for Homebased, or symptomatic and acute, thus remain in hospitals or any government facility.

Isolated cases, both asymptomatic and symptomatic undergo treatment.

The transmission dynamics are assumed to be mass action or frequency dependent.

The force of infection $\hat{\beta}=\beta e^{-c m r}$, where $\beta$ denotes the probability of contact sufficient to transmit the disease, $c$ is the quantity of oral fluid, as a result of breathing, sneezing or coughing, $m$ denotes the measure of porosity of face mask or fabric protection efficiency and $r$ is the radial distance, the of the immediate susceptible neighbour from the sick and infective person $[7,8]$.

The deaths are caused by Covid-19 only, and not by other complications.

Asymptomatic patients both unidentified and isolated at homebased care transmit disease but at a very low rate, as compared with infective class.

With the above model assumptions, variables and parameters defined, the following flow chart and subsequent 
system of differential equations represents the model.

\subsection{Model Flow Chart}

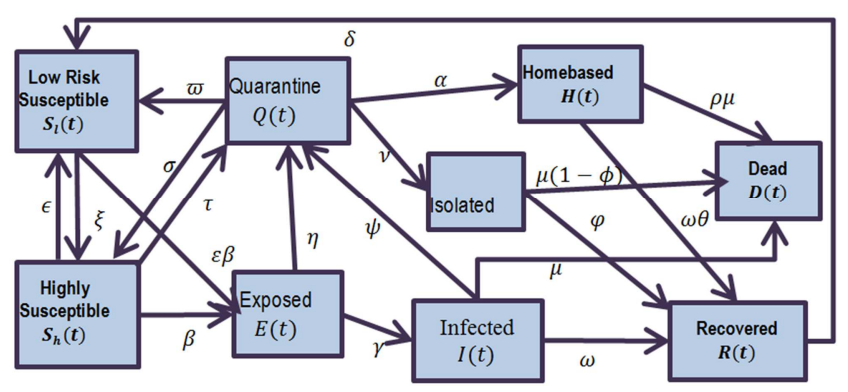

Figure 2. Covid-19 Model Flow chart with Intervention.

\subsection{Model Equations}

The following mathematical equations describe the pathways of Covid-19 disease dynamics.

$$
\begin{gathered}
\frac{d S_{h}}{d t}=\lambda-\hat{\beta} S_{h}(I+\vartheta E+\kappa H)+\sigma Q-(\epsilon+\tau) S_{h}+\xi S_{l}(5) \\
\frac{d S_{l}}{d t}=\epsilon S_{h}+\varpi Q-\varepsilon \hat{\beta} S_{l}(\vartheta E+I)-\xi S_{l}+\delta R \\
\frac{d E}{d t}=\hat{\beta} S_{h}(I+\vartheta E+\kappa H)+\varepsilon \hat{\beta} S_{l}(\vartheta E+I)-(\eta+\gamma) E \text { (7) } \\
\frac{d I}{d t}=\gamma E-(\omega+\psi+\mu) I \\
\frac{d R}{d t}=\omega I+\omega \theta H+\varphi J-\delta R \\
\frac{d Q}{d t}=\tau S_{h}+\eta E+\psi I-(\varpi+\sigma+v+\alpha) Q \\
\frac{d J}{d t}=v Q-\varphi J-\mu(1-\phi) J+\emptyset H \\
\frac{d H}{d t}=\alpha Q-\rho \mu H-\omega \theta H-\emptyset H \\
\frac{d D}{d t}=\rho \mu H+\mu(1-\phi) J+\mu I
\end{gathered}
$$

\section{Analytic Solutions}

The only rate of change of total population $\frac{d N}{d t}=\lambda$ is the constant recruitment of individuals who are tested daily. With the daily test rate of $\lambda=0.0027$, as calculated from [9], the pool of tested Susceptible increases at a constant rate.

The most important parameters in epidemiology are the basic reproductive number $R_{0}$, the contact number $\sigma$, and the replacement number $R$. Basic reproductive number $R_{0}$ is defined as the number of secondary infections that occur when one infective is introduced into a purely susceptible population. This threshold parameter [10] gives a borderline between a persistence or disease death. If $R_{0}<1$, the disease cannot replace itself and thus will die off, and if $R_{0}>1$, the disease will persist. The contact number $\sigma$ is the average number of adequate contacts of a typical infective during the entire infectious period, and the replacement number $R$ is the average number of secondary infections produced by a typical infective during the entire period of infectiousness [4].
In order to determine these parameters, the method of next generation matrix [11] is used.

The study of disease dynamics is done around the equilibrium points. These are steady state points, where the rates of change equal to zero. There are usually two equilibria, namely; disease free equilibrium (DFE) and endemic equilibrium point (EEP).

\subsection{Equilibrium Stability Analysis}

Like any other SEIR epidemiological model, we have both disease free equilibrium (DFE) and endemic equilibrium point (EEP). Define (DFE) as $E q^{0}=\left(S_{h}^{0}, S_{l}^{0}, E^{0}, I^{0}, R^{0}, Q^{0}, J^{0}, H^{0}\right)$. In absence of the disease, there is no distinction of high risk and low risk, and thus from equation (5-13), the DFE is given by; $E q^{0}=$ $\left(S^{0}, 0,0,0,0,0,0,0\right)$. This equilibrium is always stable. In the presence of the disease, the EEP denoted by $E q^{e}=$ $\left(S_{h}^{e}, S_{l}^{e}, E^{e}, I^{e}, R^{e}, Q^{e}, J^{e}, H^{e}\right)$, where

$$
\begin{gathered}
S_{h}^{e}=\frac{A+C_{1}-B}{X Y}, \mathrm{~S}_{\mathrm{l}}^{\mathrm{e}}=0, E^{e}=\frac{\xi(\omega+\psi+\mu)}{X}, I^{e}=\frac{-\varepsilon \gamma \xi}{\vartheta X}, H^{e}=\frac{\alpha b \xi \mathrm{d}}{X Y} \\
R^{e}=\frac{\varepsilon A-\epsilon B+C}{\delta X Y}, Q^{e}=\frac{\xi b \mathrm{~cd}}{X Y}, J^{e}=\frac{\xi(\alpha \epsilon+\gamma \nu)(\eta \mathrm{a}-\epsilon \gamma \psi+\gamma \tau \omega)}{X Y}
\end{gathered}
$$

where

$$
\begin{gathered}
A=\alpha \xi(\varnothing \gamma \psi \varphi+\omega \theta \mathrm{b}(\gamma \psi-\eta \mathrm{a})), B=\alpha \xi \emptyset \eta \varphi \mathrm{a}, \\
C=\xi \mathrm{c}((\gamma \omega \epsilon b \mathrm{e}+\gamma \tau \varpi \omega)-\epsilon \eta \nu \varphi \mathrm{a}+\epsilon \gamma \nu \psi \varphi), \\
C_{1}=\xi b c((\gamma \omega \mathrm{e}+\gamma \psi \varpi+\eta \varpi \mathrm{a})+\nu \varphi(\gamma \psi-\eta \mathrm{a})), \\
X=\varepsilon \beta(\gamma-\vartheta \mathrm{a}), a=(\omega+\psi+\mu), \\
b=(\varphi+\mu(1-\phi)), c=(\rho \mu+\omega \theta+\emptyset), \\
d=(\epsilon \eta a-\epsilon \gamma \psi+\gamma \tau \omega), e=(\varpi+\sigma+v+\alpha)
\end{gathered}
$$

and

$$
Y=\alpha \emptyset \tau \varphi+\mathrm{c}(\nu \tau \varphi+\tau \varpi \mathrm{b}+\epsilon b e)+\alpha \tau \omega \theta \mathrm{b}
$$

\subsection{Computation of Reproductive Ratio}

Following the next generation matrix method used in [1113], the reproductive ratio $R_{0}=\rho\left(F V^{-1}\right)$, where $F$ is the non-negative matrix of new infections and $V$ is the nonsingular matrix for the other transfer terms.

$$
R_{0}=\frac{\widehat{\beta} \vartheta S_{h}^{e}}{\eta+\gamma}+\frac{\gamma \widehat{\beta} \vartheta S_{h}^{e}}{(\eta+\gamma)(\omega+\psi+\mu)}+\frac{\alpha \kappa \widehat{\beta} \vartheta S_{h}^{e}(\gamma \psi+\eta(\omega+\psi+\mu))}{(\eta+\gamma)(\omega+\psi+\mu)(\varpi+\delta+v+\alpha)(\rho \mu+\omega \theta)}
$$

\subsection{Sensitivity Analysis of Mitigation Strategies}

Normalized sensitivity analysis of control parameters, with respect to reproductive ratio, is done using partial differentiation method defined by the vector,

$$
\tilde{E}_{p_{i}}=\frac{p_{i}}{R_{0}} \frac{\partial R_{0}}{\partial p_{i}}, i=1,2,3,
$$

where $p_{i}$ is parameter $i=1,2,3, \ldots$ of interest [14]. This method gives and normalized measure of the impact of the parameter, on the value of $R_{0}$. The desirable values of $R_{0} \leq 1$, and thus it is 
necessary to determine which of the control strategies is optimal in terms of time and cost. The dimensionless parameter $R_{0}$ as defined earlier is an important measure of the efficacy of control measures on the spread of any epidemic. As stated in [15], the sign $( \pm)$ of sensitivity index indicates the nature of the relationship, while the magnitude $|| \leq$.1 indicates the importance of the strategy in combating the spread. The parameters of interest in this model are; quarantine $(\eta, \psi)$, isolation $(v)$, testing rate $(\tau)$, homebased care $(\alpha)$, complacency $(\xi)$, public health campaign $(\epsilon)$ radial Social distance $(r)$, and fabric prevention efficiency $(m)$.

\section{Numerical Simulations}

This section presents numerical simulation results obtained using Runge-Kutta method of order 4-5, using the inbuilt MATLAB software. The parameter values were computed from real data available in [9], after 100 days of existence of
Covid-19 in Kenya, while others approximated from [1, 2, 6, 16] and are presented in the table below. The value of the mitigation parameters are to be estimated in the interval $[0,1)$.

In order to carry out simulations, it is assumed that, the populations accounted are only those captured statistically, meaning they have been tested and put in record. By the $93^{\text {rd }}$ day, total tested individuals were 130,498, with 4257 (3.26\%) confirmed cases, $117(2.75 \%)$ deaths and 1459 (34.27\%) recoveries. Out of over 40 million Kenyans, some people may have been infected by Covid-19, and some possibly recovered or died, but not captured. In the following simulation, a sample of 510,000 is taken as the initial susceptible population tested and additional tests done so as to exhaust 46 million people in a period of 150 days. The initial population is assumed to be composed of $20 \%$ high risk and $80 \%$ low risk. The following parameters used for simulation are presented in Table 2 below.

Table 2. Parameter estimates for Numerical Simulation.

\begin{tabular}{|c|c|c|c|}
\hline Symbol & Description & Value & Source \\
\hline$v$ & Proportion of positively tested people admitted to government isolation centres for treatment & 0.11 & [9] \\
\hline$\hat{\beta}$ & Probability of getting infected due to sufficient interaction with an infected person & 0.0033 & [9] \\
\hline$\epsilon$ & Change of behaviour due to public health education campaign on prevention of Covid-19 & {$[0,1]$} & Variable \\
\hline$\delta$ & Reduced rate of infection by exposed asymptomatic individuals & 0.015 & {$[17]$} \\
\hline$\sigma$ & Rate of return to high risk susceptible after testing negative & 0.9646 & Assumed \\
\hline$\mu$ & Accelerated death rate due to Covid-19 infection & 0.027 & [9] \\
\hline$\rho$ & Loss of immunity and returning to susceptible class for re-infection & 0.35 & Assumed \\
\hline$\eta$ & Transfer rate of exposed asymptomatic class for testing and quarantine (Testing rate) & 0.002744 & [9] \\
\hline$\phi$ & Effect of isolation and treatment in reducing death rate & 0.94 & Observed \\
\hline$\psi$ & Transfer rate of infected symptomatic individuals to testing and quarantine class & {$[0,1]$} & Variable \\
\hline$\theta$ & Reduction of rate of recovery due to lack of standards in home based care & 0.85 & Assumed \\
\hline$\omega$ & Recovery rate of infected individuals & 0.2992 & [9] \\
\hline$\gamma$ & Progression rate of exposed individuals to infective stage & 0.0714 & [9] \\
\hline$\xi$ & Complacency rate, where individuals revert to old normal & {$[0,1]$} & Variable \\
\hline$\vartheta$ & Modified (reduced) infective rate of exposed asymptomatic individuals & 0.5 & Assumed \\
\hline$\alpha$ & Transfer rate of infected people from quarantine to homebased isolation and care & 0.89 & [9] \\
\hline$\kappa$ & Modification of infectivity by homebased infected individuals & 0.315 & Calculated \\
\hline$\partial$ & Rate of transfer of home based to Isolation & 0.2 & {$[0,1]$} \\
\hline$\varpi$ & Transfer of individuals to low risk after testing negative & 0.7 & Calculated \\
\hline$c$ & Human aerosol plume dynamics and viral particles transmissivity constant in $\%$ & {$[11.3,89.6]$} & {$[7,8]$} \\
\hline$m$ & Face mask Porosity & $<0.896$ & {$[0,1]$} \\
\hline$d$ & Social distance observed between an infective and a susceptible individual & Variable & {$[0, \infty)$} \\
\hline
\end{tabular}

\subsection{Simulation of Covid-19 in Absence of Intervention}
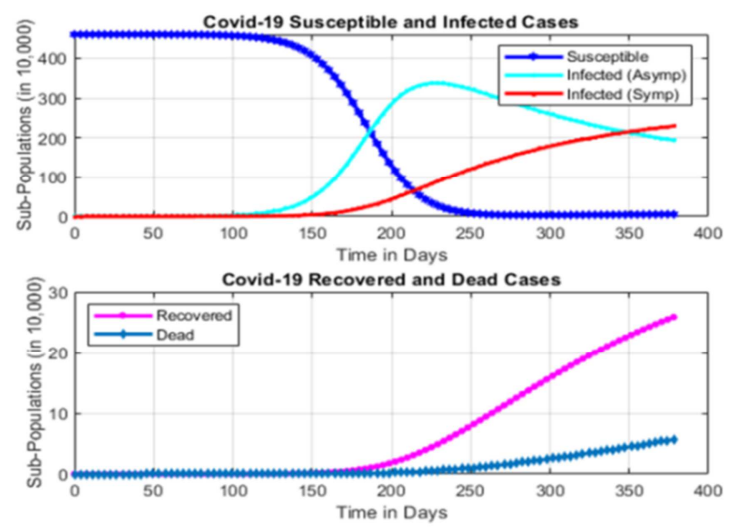

Figure 3. Covid-19 Dynamics in Absence of Intervention.
In absence of intervention, the model reduces SEIR model represented by equation (1 - 4). In absence of intervention, the effects of public health campaign, treatment, isolation, home based care, travelling ban, use of masks, and therefore all people are considered high risk, and there will be only one category of susceptible. Setting the relevant parameters equal to zero, we obtain the results in Figure 3 below.

From Figure 3, it is clear that, by the end of 8 months (250 days), all the susceptible population will have been infected, with a few recovering $(26,000)$ and deaths rising up to about 70,000. This is catastrophic and such an event cannot happen because of parasympathetic human response to protect himself. However, the disease dies off from the $225^{\text {th }}$ day. 


\subsection{Sensitivity Analysis of Intervention Strategies}

In order to guide on which intervention strategy is more effective, sensitivity on the reproductive ratio is computed.
Using the method defined in Section 3.3, the sensitivity of each intervention strategy, in achieving the condition $R_{0} \leq 1$ is presented in Figure 4 below.

Table 3. Comparative Sensitivity analysis of mitigation strategies.

\begin{tabular}{llll}
\hline Parameter & Definition & Relationship & Elasticity \\
\hline$\alpha$ & Proportion of individuals at homebased care & Direct & 2.5681 \\
$\psi$ & Transfer rate of individuals in Infective class to quarantine & Inverse & -1.6237 \\
$\eta$ & Transfer rate of exposed asymptomatic individuals to quarantine & Inverse & -1.7701 \\
$v$ & Transfer rate of confirmed cases to government isolation center & Inverse & -6.4162 \\
$\hat{\beta}$ & Probability of getting infected & Direct & 2.2483 \\
$r$ & Radial distance between infective and susceptible & Inverse & 0.9966 \\
$m$ & Efficacy of the face mask (Porosity, correct use, fabric quality) & Inverse & -0.8857 \\
\hline
\end{tabular}

The values in Table 3. Above indicates that, the most responsive parameter to be addressed is proportion of homebased care infected patients and government facility care center proportions, These are followed by quarantine rate. The choice of the method to be used depends on associated cost. Also the use of public health campaign and guarding against complacency cannot be ignored. The graphs of parameter elasticities are shown in Figure 4.

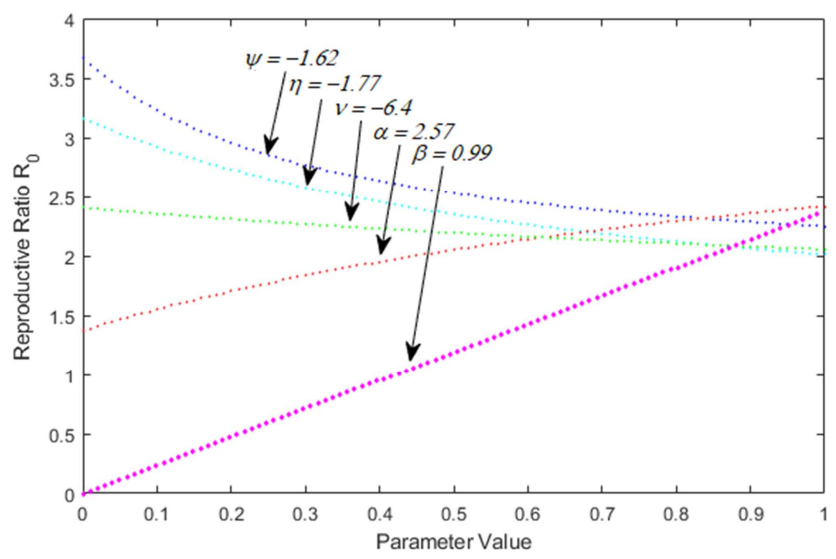

Figure 4. Elasticity graphs of Covid-19 Intervention Parameters.

\subsection{Social Distance and Face mask Efficiency}

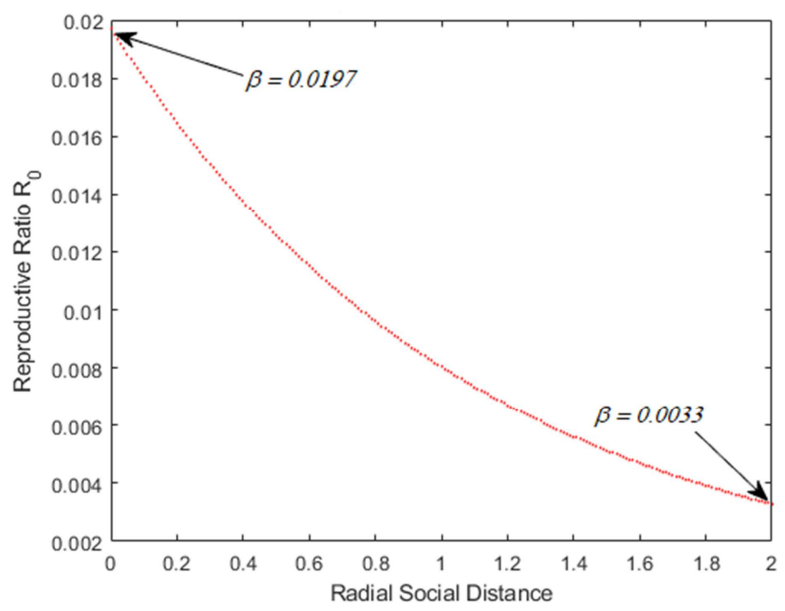

Figure 5. Effects of social distance on Viral Infectivity.

Efficiency of face masks in protecting the transmission of virus from an infectious person to a susceptible depends on various factors. These factors can be classified into three, namely; the human factors, environmental factors and the face mask factors. Human factors include the viral load or concentration, amount of aerosol, and air flow dynamics. Environmental include humidity, wind speed, room ventilation, and nearness of people. The face mask factors include fabric quality, proper use, frequency of replacement, and size or area of coverage of face mask [7, 8]. In this paper, combined effect of social distance and fabric quality are jointly analyzed in the parameter $\hat{\beta}=\beta e^{-c m r}$.

From sensitivity analysis in Table 3 ., the social distance has much more effect in reducing the force of infection from $\beta=0.0197$ to $\beta=0.0033$, as shown in Figure 5 . above. This will consequently reduce the basic reproductive ratio from $R_{0}=14.0362$ to $R_{0}=2.3388$, a $83.34 \%$ decrease. The combined face mask and social distance intervention translates to a reduction of infected people (in 100 days of the pandemic) from $E_{100}=451,476$ (in absence of face mask and social distance strategy), to $E_{100}=2,859$, at $89.6 \%$ observance of the control requirement.

\subsection{Simulation Results}
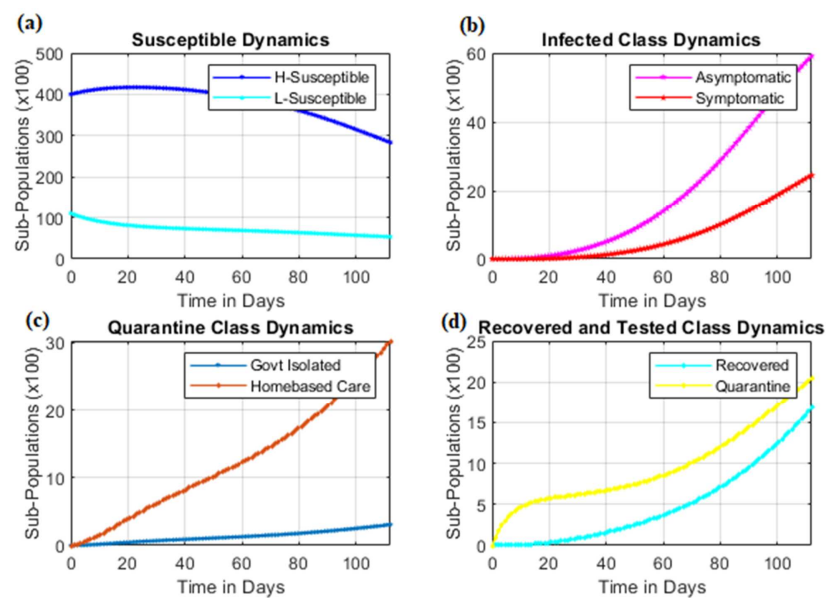

Figure 6. Simulation Results for Covid-19 dynamics in the first 100 days.

Using the parameter values in Table 2. together with intervention strategies in Table 3., we obtain a scenario depicted in Figure 6, and Figure 7 below. This is comparable with exact statistics by WHO [9].

Clearly, Figure 6 shows that as the disease spreads, the 
number of susceptible in Figure 6 (a) decreases as the number of infected (both symptomatic and asymptomatic) in Figure 6 (b) increases. Meanwhile, intervention strategies will witness increased number of isolated cases Figure 6 (c) and the same trend evidenced in the number of recoveries and quarantine cases Figure 6 (d). The same scenario is evidenced in Kenya, as per WHO statistics [9]. A comparison of simulation results and Kenyan - WHO data are presented in Figure 7 below.

The simulated results at day 100 of infected, recovered and deaths are 5,399, 1,466 and 118 respectively as compared to WHO statistics of 4,257, 1,459 and 117 respectively. This shows a disparity of infected class by 1,142 . This disparity could be associated with asymptomatic cases who are not tested and therefore not captured.
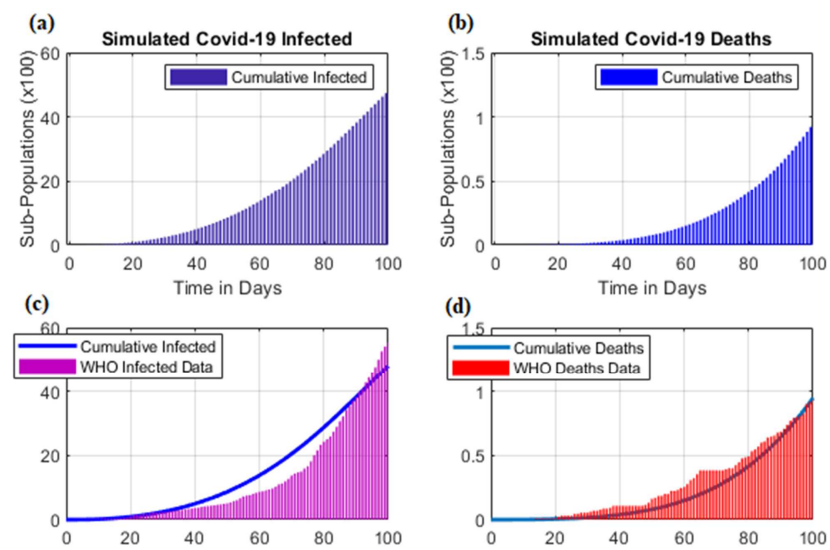

Figure 7. Simulation results compared with WHO Statistics for Covid-19 Cumulative Infected and Deaths in 100 days.

\section{Conclusion}

Covid-19 pandemic has been the worst viral disease whose effect has been felt in the whole world. Businesses closed, schools closed, travel ban put in place, curfew, regulations on limitation of social interaction among other containment measures. In this paper, analysis on the sensitivity of intervention parameters indicated that isolation of infectives to government isolation centers is the most effective strategy, This requires mass testing, in order to identify and isolate infected individuals. This however is costly and overwhelms the existing limited facilities. The second best strategy is the use of social distance and face mask. A combination of all these strategies will have a synergetic impact on the containment of Covid-19 pandemic. The face mask however should not be just any, but an effective face mask, which can reduce transmissivity of viral particles. These strategies can help buy time as the search for a vaccine is in progress.

\section{References}

[1] Millán-Oñate, J., et al., A new emerging zoonotic virus of concern: the 2019 novel Coronavirus (COVID-19). Infectio, 2020. 24 (3).
[2] Read, M. C., Severe respiratory disease associated with a novel coronavirus, 19 February 2013 (ECDC, RRA, edited). 2020.

[3] Weiss, S. R. and S. Navas-Martin, Coronavirus pathogenesis and the emerging pathogen severe acute respiratory syndrome coronavirus. Microbiol. Mol. Biol. Rev., 2005. 69 (4): p. 635664.

[4] Keeling, M. J. and P. Rohani, Modeling infectious diseases in humans and animals. 2011: Princeton University Press.

[5] Rodrigues, H. S., Optimal control and numerical optimization applied to epidemiological models. arXiv preprint arXiv: 1401.7390, 2014.

[6] Sohrabi, C., et al., World Health Organization declares global emergency: A review of the 2019 novel coronavirus (COVID19). International Journal of Surgery, 2020.

[7] Tang, J. W., et al., Airflow dynamics of human jets: sneezing and breathing-potential sources of infectious aerosols. PLoS One, 2013.8 (4): p. e59970.

[8] Bowen, L. E., Does that face mask really protect you? Applied biosafety, 2010. 15 (2): p. 67-71.

[9] Organization, W. H., Coronavirus disease 2019 ( COVID-19): situation report, 93. 2020.

[10] Kermack, W. O. and A. G. McKendrick, A contribution to the mathematical theory of epidemics, in Royal Soc. 1927: London (A). p. 700-721.

[11] Van den Driessche, P. and J. Watmough, Reproduction numbers and sub-threshold endemic equilibria for compartmental models of disease transmission. Mathematical biosciences, 2002. 180 (1-2): p. 29-48.

[12] Podder, C. N., et al., Mathematical study of the impact of quarantine, isolation and vaccination in curtailing an epidemic. Journal of Biological Systems, 2007. 15 (02): p. 185-202.

[13] Yan, X. and Y. Zou, Control of epidemics by quarantine and isolation strategies in highly mobile populations. International Journal of Information and Systems Sciences, 2009. 5 (3-4): p. 271-286.

[14] Samsuzzoha, M., M. Singh, and D. Lucy, Uncertainty and sensitivity analysis of the basic reproduction number of a vaccinated epidemic model of influenza. Applied Mathematical Modelling, 2013. 37 (3): p. 903-915.

[15] Blower, S. M. and H. Dowlatabadi, Sensitivity and uncertainty analysis of complex models of disease transmission: an HIV model, as an example. International Statistical Review/Revue Internationale de Statistique, 1994: p. 229-243.

[16] Stoecklin, S. B., et al., First cases of coronavirus disease 2019 (COVID-19) in France: surveillance, investigations and control measures, January 2020. Eurosurveillance, 2020. 25 (6): p. 2000094.

[17] Bai, Y., X. Nie, and C. Wen, Epidemic prediction of 2019$\mathrm{nCoV}$ in Hubei province and comparison with SARS in Guangdong province. Available at SSRN 3531427, 2020. 